\title{
Stem cell therapy: in treatment of neurodegenerative diseases
}

\begin{abstract}
Over the past 25 years, stem cell therapies have become an attractive field to treat and investigate neurodegenerative diseases. Stem cells are undifferentiated cells with the ability of proliferation, regeneration, differentiation in other cells. Stem cells are divided into two categories of embryonic and adult. In some other classification stem cells are divided into Totipotent, Multipotent and Unipotent cells. Stem cell therapy used in different diseases like neurodegenerative disease, inflammatory bowel disease, liver disease, diabetes, heart disease, bone disease, renal disease, chronic wounds, graft-versus-host disease, sepsis, lymphoblastic leukemia, myeloid leukemia, thalassemia, multiple myeloma, cycle cell anemia and respiratory diseases. In the current review, we detail the current state of stem cell research in neurodegenerative diseases, with a brief introduction about stem cell therapy. We also discuss the hurdles associated with stem cell therapies from bench to bedside. In this review, the final goal is the evaluation of cell therapy in the treatment of different neurodegenerative diseases like Parkinson's disease, Huntington's disease, Amyotrophic lateral sclerosis, Alzheimer, Spinal muscular atrophy, Stroke and the burden of these diseases on the society underline the need for the practice of stem cell therapies.
\end{abstract}

Keywords: stem cell, neurodegenerative disease, neurons, neurological disorders
Volume 4 Issue I - 2018

\author{
Sameer K Varma,' Mohsina Hyder K, ${ }^{2}$ Swati \\ Som, ${ }^{2}$ SP Dhanabal ${ }^{3}$ \\ 'Department of Pharmaceutical Biotechnology, \\ Shivarathreeswara University, India \\ ${ }^{2}$ Department of Pharmacy Practice, Shivarathreeswara \\ University, India \\ ${ }^{3}$ Department of Pharmacognosy \& Phytopharmacy, \\ Shivarathreeswara University, India
}

\begin{abstract}
Correspondence: Sameer K Varma, Department of Pharmaceutical Biotechnology, JSS College of Pharmacy, Rocklands, Ooty, Tamil Nadu, India, Tel +9184897846/5,
\end{abstract} Email sameer.varma22@gmail.com

Received: July 24, 2017 | Published: February 01, 2018
Abbreviations: ND: neurodegenerative disease; PD, parkinson's disease; AD, alzheimer disease; ALS, amyotrophic lateral sclerosis; ES, embryonic stem; iPS, induced pluripotent stem; MSCs, mesenchymal stem cells; SOD1, superoxide dismutase 1; SMA, spinal muscular atrophy

\section{Introduction}

Stem cells are undifferentiated cells that have the ability of proliferation, regeneration, conversion to differentiated cells and tissue production. Regeneration means that these cell shave the ability of asymmetric division, which one of the resulting cells remains as stem cell while another cell, which is called daughter cell, becomes one cell of germ layer. Stem cells may remain inactive for a long time till they enter cell division again. ${ }^{1,2}$ Stem cells are divided into two groups: embryonic and adult stem cells. Embryonic stem cells are derived from the zygote cell which is fertilized in vitro and usually is 4-5days embryo that is in the form of a hollow ball called blastocyst. A Blastocyst is composed of three parts: the trophoblast layer that is surrounding blastocyst, a hollow cavity inside the blastocyst and inner cell mass that changes in the embryo. Since zygote cells can differentiate into placenta and fetal cells, sometimes they are considered as the only true totipotent stem cells. Because the inner cell mass of the blastocyst does not have the ability to differentiate into placenta cells, it is called the pluripotent cell. Non-differentiated cells other than embryonic stem cells can be found in differentiated cells of specific tissues after birth. These cells are called adult or nonembryonic stem cells, but a more accurate word for them is "somatic stem cells" because these cells also exist in children and umbilical cord. They are divided into two main categories: hematopoietic stem cells that can differentiate into blood cells and mesenchymal stem cells that are less differentiated. Nose, muscle, liver, skin, brain, retina and limbus of the eye are the other sources of adult stem cells. One of the most important advantages of adult stem cells over embryonic stem cells is because of the fact that they can be obtained without the need for destruction of embryo. ${ }^{3}$ Different types of stem cells are shown in Figure 1. The pluripotent stem cell differentiates into the multipotent cell of 3 different germ layers (ectoderm, mesoderm and endoderm layer). The multipotent cell differentiates into unipotent cell of a specific cell lineage within its germ layer. If differentiation process is successful, the resulting cells will be called as progenitor cells or stem cell-like cells that have the capability of regeneration. ${ }^{4-6}$ Stem cell therapy has been evaluated in various blood diseases (such as lymphoblastic leukemia, myeloid leukemia, thalassemia, multiple myeloma, cell cycle anemia). The aim of this review is to evaluate cell therapy in different diseases.

\section{Stem cell therapy}

Stem cells have the capacity to proliferate and differentiate into multiple cellular lineages. There are different classifications of stem cells that reflect the range of possible cell types they can produce, and the way in which stem cells are derived. These include embryonic stem (ES) cells; progenitor cells, mesenchymal stem cells (MSCs), and induced pluripotent stem (iPS) cells. ${ }^{7}$ To appreciate the potential applications of stem cell technology to neurodegenerative diseases, it is important to understand the characteristics of the various stem cell types available and the potential impact of cellular therapies on disease mechanisms. 


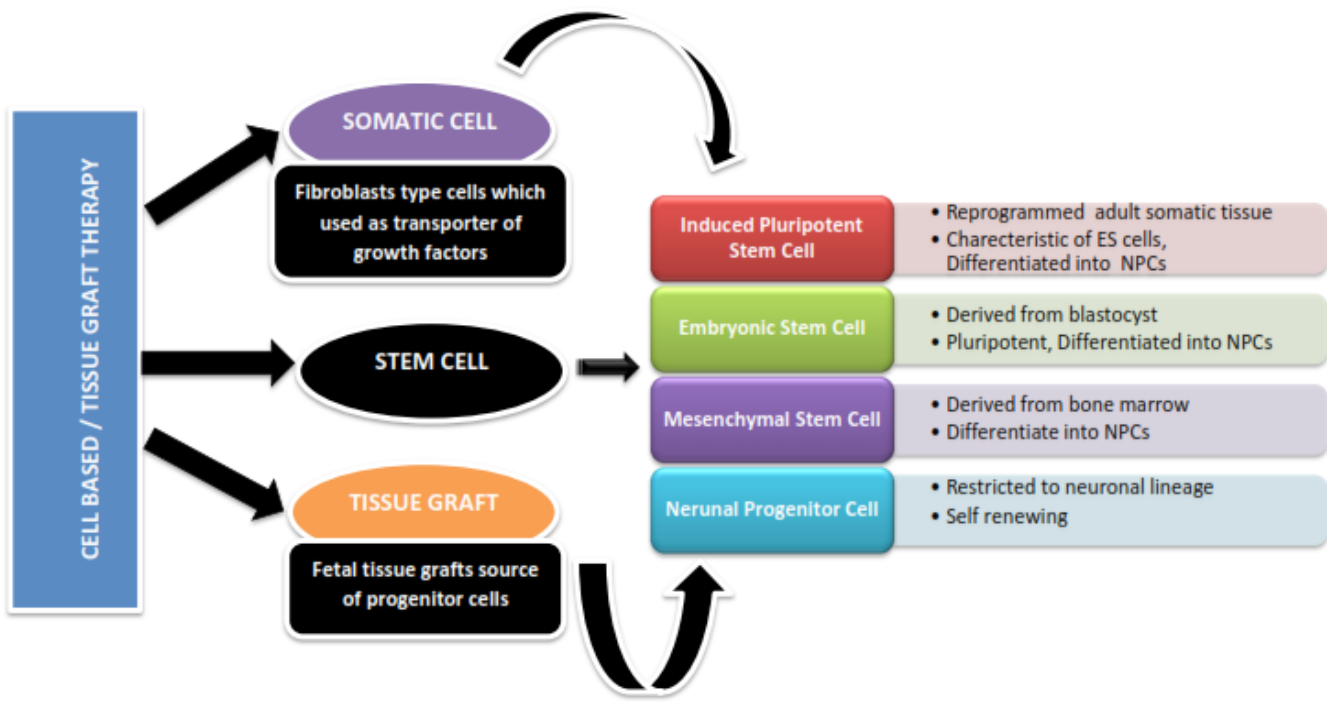

Figure I Stem cell therapy and classification.

\section{Cellular therapy for neurodegenerative diseases}

Neurodegenerative diseases are characterized by the loss of neurons in the brain or spinal cord. Acute neurodegeneration may result from a temporally discrete insult, such as stroke or trauma, leading to a localized loss of neurons at the site of injury. Chronic neurodegeneration may develop over a long period of time and results in the loss of a particular neuronal subtype or generalized loss of neuronal populations. In the brain, Alzheimer's disease (AD) and Huntington's disease (HD) result in widespread loss of neurons, while Parkinson's disease (PD) involves the specific and localized loss of dopaminergic (DA) neurons in the substantia nigra. In the brainstem and spinal cord, amyotrophic lateral sclerosis (ALS) and spinal muscular atrophy (SMA) involve the degeneration and loss of motor neurons (MNs) ${ }^{8,9}$ While this condition all exhibit unique neuronal pathologies, the exact mechanisms for neuronal loss are complex, making the identification of efficacious treatments elusive. Neurodegenerative diseases create a tremendous burden on society and despite decades of research, effective treatments do not exist. Cellular therapies are attractive options and the application of stem cell research to neurodegenerative diseases is rapidly expanding.

\section{Alzheimer's disease}

$\mathrm{AD}$ is the most frequent form of dementia; it is characterized by memory loss and cognitivedecline. As the disease progresses, there is a widespread synaptic loss throughout the brain, involving the basal forebrain cholinergic system, amygdala, hippocampus and several cortical areas. There is an increased risk of developing $\mathrm{AD}$ with age, and the majority of $\mathrm{AD}$ cases are late-onset, developing over 65 years of age. Current treatment options for $\mathrm{AD}$ are centered on regulating neurotransmitter activity, such as treatment with acetylcholinesterase inhibitors to enhance cholinergic function, provide only partial and temporary alleviation of symptoms. Targeting the cholinergic system using stem cell therapies may provide environmental enrichment. ${ }^{10}$ Neurogenesis in the hippocampus decreases as we age and is exacerbated in $\mathrm{AD}$, therefore cellular therapies which enhance neurogenesis or replace lost neurons may also delay the progression of AD. Some studies showed that, the mesenchymal stem cells derived from human umbilical cord injected into Alzheimer's mice and observed that markers of glial activity, oxidative stress and apoptosis were decreased in mouse brain. Also cognitive abilities and learning and memory in mice were returned. ${ }^{11}$ The corpus callosum is another structure that is affected early in Alzheimer's disease. Some study demonstrates the feasibility of targeting the corpus callosum and identifies an effective immunosuppression regimen for transplanted neural stem cell survival, results supported further preclinical development of the corpus callosum as a therapeutic target in Alzheimer's disease. ${ }^{12}$

\section{Parkinson's disease}

PD is the second most common neurodegenerative disease, after $\mathrm{AD}$, and characterized by progressive destruction of dopaminergic neurons in substantia nigra of midbrain, that is motor signs such as bradykinesia, rigidity; akinesis, stiffness and rest tremor are due to destruction of terminal dopaminergic neurons in basal ganglia including caudate nucleus and putamen which results in balance disorders. The Pathological hallmarks of PD are characterized by the presence of Lewy bodies composed of alpha-synuclei ( $\alpha$-syn) protein beyond the nigra and the cortex. Levodopa can improve the symptoms, but it cannot prevent neurons from destruction. Embryonic stem cells have the ability of differentiation of neural stem cells and subsequently dopaminergic neurons, but they have short survival time. Some research showed that combination of Noggin that is a bone morphogenic protein antagonist with stromal cells can increase production of progenitor neouroepithelial cells that have e potential of differentiating to dopaminergic neurons. ${ }^{13}$ Mutations in genes such as SNCA cause PD in an autosomal dominant manner and patients have motor and non-motor symptoms that are typical for sporadic PD. ${ }^{14}$ Mesenchymal cells injected into inferolateral ventricular area in parkinson patients and observed significant improvement in symptoms such as facial gestures, gait and freezing episode. ${ }^{15}$

\section{Huntington's disease}

HD is a fatal, intractable, autosomal dominant polyglutamine disease caused by the accumulation of CAG repeats in the huntingtin gene. Mutations in the huntingtin gene (HTT) lead to polyglutamine 
repetitions, causing psychiatric and physiologic alterations like dementia, personality changes, involuntary motor activity, and cognitive impairment associated with the progressive loss of medium spiny neurons (MSNs) are the main characteristics of HD. ${ }^{16}$ Loss of GAB Aergic neurons in the striatum is also accompanied by degeneration in the cortex, brain stem and hippocampus. There are the main strategies for the treatment of HD, one strategy is to enhance neurogenesis and the other one is a cell therapy to protect neuronal cell populations or to replace dysfunctional or dying cells. Cells from HD patients were first reprogrammed into iPSC in $2010,{ }^{17}$ and alterations in electrophysiology, cell metabolism, adherence and toxicity were reported. Expansion of a CAG repeat alters the transport and release of BDFN and increases glutamate receptors, producing toxicity and oxidative stress in neuron and glial cells. ${ }^{18,19}$ Stem-cell therapy aims to restore or preserve brain function by replacing and protecting striatal neurons. At this time, using stem cells from the delivery of trophic factors and neuroprotection to prevent disease progression seems a more achievable clinical goal in HD than neuronal replacement.

\section{Amyotrophic lateral sclerosis}

ALS is an adult-onset fatal devastating neurodegenerative disorder that is characterized by progressive destruction of neurons of spinal cord and motor neurons of cortical brain ${ }^{20}$ leading to fatal paralysis. Patients present with loss of coordination and muscle strength with transition to complete loss of muscle control. Death typically results from respiratory failure within 2-5years of diagnosis. Multiple cell types and mechanisms are likely involved in ALS pathogenesis, which makes the development of conventional drug therapies difficult. The cause of ALS is not yet elucidated, however, mutations in genes SOD1, C9orf72, TDP-43, FUS/TLS, angiogenin, Matrin $3^{21,22}$ and others, have been associated with ALS. A stem-cell therapy could restore or preserve the function of both upper and lower motor neurons, ${ }^{23,24}$ and new neurons could become integrated into existing neural circuitries. ${ }^{25,26}$ Many studies have indicated that it is possible to generate motor neurons in culture from stem cells that include ESCs and NSCs. Mouse ESC-derived motor neurons transplanted into motor neuron injured rat spinal cord survived and extended axons into ventral root, and human ESCs transplantedinto cerebrospinal fluid of rats with motor neuron injury migrated into the spinal cord and led to improved motor function. ${ }^{27-29}$

\section{Stroke}

A stroke causes loss of a large number of neurons and glial cells. Therapies aimed at improving cerebral blood flow; there has been increasing emphasis on neuroprotective strategies. Cell therapy opens up new horizons in the treatment of this disease through facilitation of neuronal regeneration process. Animal studies and several preclinical trials confirm efficacy of cell therapy in functional improvement after stroke. Protection of neurons, regulation of immune system, increase of internal healing processes, vascular regeneration and stimulation of host brain plasticity and use of internal progenitors are its possible effects. ${ }^{30-33}$ A variety of cell types have been tried for restoration of brain function after stroke, mostly in rodent models. The technical and ethical difficulties associated with these cells promoted a search for alternatives. Autologous somatic stem cells are a very attractive source; graft rejection is not an issue. Cerebral ischemia activates endogenous reparative processes, such as increased proliferation of neural stem cells (NSCs) in the subventricular zone (SVZ) and migration of neural progenitor cells (NPCs) toward the ischemic area. In this study data demonstrate the neurodegenerative activity of
MANF that facilitates differentiation and migration of NPCs, thereby increasing recruitment of neuroblasts in stroke cortex. ${ }^{34}$

\section{Spinal muscular atrophy}

SMA is an autosomal recessive neurodegenerative disease caused by mutations in survival of motor neuron gene $(S M N-1)$, characterized by a selective and progressive lossof lower motor neurons resulting in degeneration of motor neurons in the spinal cord and muscular atrophy on limbs and trunk. ${ }^{35,36}$ In humans, low levels of SMN protein may be produced by alternative splicing variants encoded by the $S M N 2$ gene. Current pharmaceutical developments and gene therapy treatments focus on regulating SMN2 to treat SMA. Cellular therapies, however, have been examined in mouse models of SMA, where grafting of ES derived NPCs protected MNs from degeneration and improved survival. ${ }^{37}$ The correction of $S M N$ gene, using singlestranded oligonucleotide, was shown to restore the $S M N$ gene profile in neurons derived from SMA-iPSC, converting SMN2 in SMN1. ${ }^{38}$ It is possible that for SMA, transient rescue of the developmental loss of SMN may be sufficient to confer efficacy, which may not be the case for other neurodegenerative diseases where long-term degeneration of the transplanted cells is a valid concern.

\section{Conclusion}

This review discusses the path for disease, treatment and prevention is through the unveiling of pathogenesis and physiological mechanisms that ultimately result in the phenotypic symptoms of diseases. Major issues associated with stem cell therapy by transplantation for neurodegenerative diseases. Importantly, these models might offer advantages in various aspects discussed along the text. Moreover, they complement in vivo studies that investigate the mechanisms involved in the pathogenesis of neurodegeneration. Stem cells from a variety of sources have shown effectiveness in improving motor function after neurodegenerative diseases in animal experiments and clinical trials.

\section{Acknowledgements}

None.

\section{Conflict of interest}

The author declares no conflict of interest.

\section{References}

1. Orford KW, Scadden DT. Deconstructing stem cell self-renewal: genetic insights into cell-cycle regulation. Nat Rev Genet. 2008;9(2):115-128.

2. Evans MJ, Kaufman MH. Establishment in culture of pluripotential cells from mouse embryos. Nature. 1981;292(5819):154-156.

3. Cui YF, Hargus G, Xu JC, et al. Embryonic stem cell-derived L1 overexpressing neural aggregates enhance recovery in Parkinsonian mice. Brain. 2010;133(Pt 1):189-204.

4. Tuch BE. Stem cells: a clinical update. Aust Fam Physician. 2006;35(9):719-721.

5. Appel SH, Engelhardt JI, Henkel JS, et al. Hematopoietic stem cell transplantation in patients with sporadic amyotrophic lateral sclerosis. Neurology. 2008;71(17):1326-1334.

6. Takahashi K, Yamanaka S. Induction of pluripotent stem cells from mouse embryonic and adult fibroblast cultures by defined factors. Cell. 2006;126(4):663-676. 
7. Xu X, Lei Y, Luo J, et al. Prevention of $\beta$-amyloid induced toxicity in human iPS cell-derived neurons by inhibition of Cyclin-dependent kinases and associated cell cycle events. Stem Cell Res. 2013;10(2):213227.

8. Soldner F, Laganiere J, Cheng AW, et al. Generation of isogenic pluripotent stem cells differing exclusively at two early onset Parkinson point mutations. Cell. 2011;146(2):318-331.

9. Zimprich A, Biskup S, Leitner P, et al. Mutations in LRRK2 cause autosomal-dominant parkinsonism with pleomorphic pathology. Neuron 2004;44(4):601-607.

10. Wolfe MS. When loss is gain: reduced presenilin proteolytic function leads to increased Abeta42/Abeta40. Talking Point on the role of presenilin mutations in Alzheimer disease. EMBO Rep. 2007;8(2):136-140.

11. Lee HJ, Lee JK, Lee H, et al. The therapeutic potential of human umbilical cord blood-derived mesenchymal stem cells in Alzheimer's disease. Neurosci Lett. 2010;481(1):30-35.

12. McGinley LM, Kashlan ON, Chen KS, et al. Human neural stem cel transplantation into the corpus callosum of Alzheimer's mice. Ann Clin Transl Neurol. 2017;4(10):749-755.

13. Sonntag KC, Pruszak J, Yoshizaki T, et al. Enhanced Yield of Neuroepithelial Precursors and Midbrain-Like Dopaminergic Neurons from Human Embryonic Stem Cells Using the Bone Morphogenic Protein Antagonist Noggin. Stem Cells. 2007;25(2):411-418.

14. Singh Dolt K, Hammachi F, Kunath T. Modeling Parkinson's disease with induced pluripotent stem cells harboring alpha-synuclein mutations. Brain Pathol. 2017;27(4):545-551.

15. Venkataramana NK, Kumar SK, Balaraju S, et al. Open-labeled study of unilateral autologous bone-marrow-derived mesenchymal stem cel transplantation in Parkinson's disease. Transl Res. 2010;155(2):62-70.

16. Sawa A, Tomoda T, Bae BI. Mechanisms of neuronal cell death in Huntington's disease. Cytogenet Genome Res. 2003;100(1-4):287-295.

17. Ross CA, Tabrizi SJ. Huntington's disease: from molecular pathogenesis to clinical treatment. Lancet Neurol. 2011;10(1):83-98.

18. Zhang N, An MC, Montoro D, et al. Characterization of Human Huntington's Disease Cell Model from Induced Pluripotent Stem Cells. PLoS Curr. 2010;2:1193.

19. Shin JY, Fang ZH, Yu ZX, et al. Expression of mutant huntingtin in glial cells contributes to neuronal excitotoxicity. J Cell Biol. 2005;171(6):1001-1012.

20. Deda H, Inci MC, Kürekçi AE, et al. Treatment of amyotrophic lateral sclerosis patients by autologous bone marrow-derived hematopoietic stem cell transplantation:a 1-year follow-up. Cytotherapy. 2009;11(1):18-25.

21. Pasinelli P, Brown RH. Molecular biology of amyotrophic lateral sclerosis: insights from genetics. Nat Rev Neurosci. 2006;7(9):710-723.
22. Da Cruz S, Cleveland DW. Understanding the role of TDP -43 and FUS/ TLS in ALS and beyond. Curr Opin Neurobiol. 2011;21(6):904-919.

23. Padhi AK, Jayaram B, Gomes J. Prediction of functional loss of human angiogenin mutants associated with ALS by molecular dynamics simulations. Sci Rep. 2013;3:1225.

24. Johnson JO, Pioro EP, Boehringer A, et al. Mutations in the Matrin 3 gene cause familial amyotrophic lateral sclerosis. Nat Neurosci. 2014;17(5):664-666.

25. Silani V, Calzarossa C, Cova L, et al. Stem cells in amyotrophic lateral sclerosis: motor neuron protection or replacement? CNS Neurol Disord Drug Target. 2010;9(3):314-324.

26. Silani, V, Cova L, Corbo M, et al. Stem-cell therapy for amyotrophic lateral sclerosis. Lancet. 2004;364:200-202.

27. Yang EJ, Jiang JH, Lee SM, et al. Electroacupuncture reduces neuroinflammatory responses in symptomatic amyotrophic lateral sclerosis model. J Neuroimmunol. 2010;223(1-2):84-91.

28. Thonhoff JR, Ojeda L, Wu P. Stem cell-derived motor neurons: applications and challenges in amyotrophic lateral sclerosis. Curr Stem Cell Res Ther. 2009;4(3):178-199.

29. Lepore AC, Maragakis NJ. Targeted stem cell transplantation strategies in ALS. Neurochem Int. 2007;50(7-8):966-975.

30. Savitz SI, Rosenbaum DM, Dinsmore JH, et al. Cell transplantation for stroke. Ann Neurol. 2002;52(3):266-275.

31. Cui X, Chopp M, Zacharek A, et al. Chemokine, vascular and therapeutic effects of combination Simvastatin and BMSC treatment of stroke. Neurobiol Dis. 2009;36(1):35-41.

32. Liu Z, Li Y, Zhang X, et al. Contralesional axonal remodeling of the corticospinal system in adult rats after stroke and bone marrow stromal cell treatment. Stroke. 2008;39(9):2571-2577.

33. Corti S, Nizzardo M, Nardini M, et al. Embryonic stem cell-derived neural stem cells improve spinal muscular atrophy phenotype in mice. Brain. 2010;133(2):465-481.

34. Tseng, Anttila JE, Khodosevich K, et al. MANF Promotes Differentiation and Migration of Neural Progenitor Cells with Potential Neural Regenerative Effects in Stroke. Mol Ther. 2017;26(1):238-255.

35. Ebert AD, Yu J, Rose FF Jr, et al. Induced pluripotent stem cells from a spinal muscular atrophy patient. Nature. 2009;457:277-280.

36. Wu CY, Whye D, Glazewski L, et al. Proteomic assessment of a cell model of spinal muscular atrophy. BMC Neurosci. 2011;12:25.

37. Corti S, Nizzardo M, Nardini M, et al. Neural stem cell transplantation can ameliorate the phenotype of a mouse model of spinal muscular atrophy. J Clin Invest. 2008;118(10):3316-3330.

38. Corti S, Nizzardo M, Simone C, et al. Genetic correction of humaninduced pluripotent stem cells from patients with spinal muscularatrophy. Sci Transl Med 2012:4:165. 